\title{
Prooxidant activity and toxicity of nordihydroguaiaretic acid in clone-9 rat hepatocyte cultures
}

\author{
Saura C. Sahu ${ }^{\mathrm{a}, *}$, Dennis I. Ruggles ${ }^{\mathrm{b}}$, Michael W. O’Donnell ${ }^{\mathrm{b}}$ \\ a Division of Toxicology, Office of Applied Research and Safety Assessment, Center for Food Safety and Applied Nutrition, \\ US Food and Drug Administration, 8301 Muirkirk Road, Laurel, MD 20708, USA \\ ${ }^{\mathrm{b}}$ Division of Mathematics, Office of Scientific Analysis and Support, Center for Food Safety and Applied Nutrition, US Food and Drug Administration, \\ 8301 Muirkirk Road, Laurel, MD 20708, USA
}

Received 28 December 2005; accepted 24 May 2006

\begin{abstract}
Nordihydroguaiaretic acid (NDGA) is a polyphenol. It is present at high concentrations in the leaves of the evergreen desert shrub, Larrea tridentate (Creosote bush), which has a long history of medicinal use traditionally by the native Americans and Mexicans. It is generally believed that the antioxidant properties of NDGA are responsible for the medicinal value of this desert shrub.

The clone-9 rat hepatocyte cultures were used as an in vitro model to assess the hepatotoxic potential of NDGA and to determine whether it exhibits any prooxidant activity. The hepatocyte cultures were treated with NDGA for $2 \mathrm{~h}$ at $37^{\circ} \mathrm{C}$ at concentrations of $0-100 \mu \mathrm{M}$. After the treatment period the cells, the culture supernatants and cell lysates were assayed for evaluation of prooxidant activity and toxicity of NDGA. Oxidative stress level and oxidative cell injury as measured by the peroxidation of membrane lipids and DNA double-strand breaks were used to index prooxidant activity. Cytotoxicity as measured by the leakage of the liver enzyme lactate dehydrogenase (LDH) into the culture medium, mitochondrial function and extent of cell proliferation were used as the endpoints of toxicity. Significant concentration-dependent differences were observed in these biomarkers over the concentration range examined demonstrating the prooxidant activity and toxicity of NDGA in clone-9 rat hepatocyte cultures.
\end{abstract}

Published by Elsevier Ltd.

Keywords: Hepatotoxicity; Liver toxicity; Hepatocytes; Clone-9 cells; NDGA

\section{Introduction}

Nordihydroguaiaretic acid (NDGA) is a polyphenol. It is present at high concentrations in the leaves of the evergreen desert shrub Creosote bush, Larrea tridentata, which grows throughout much of the southwest USA. This shrub has a long history of medicinal use for a variety of health problems (Arteaga et al., 2005) traditionally by the native Americans and Mexicans. This polyphenol is present in the herbal product "Chaparral" prepared from the leaves of this desert shrub. Chaparral is used as a dietary supplement in the form of tea, capsules or tablets.

\footnotetext{
* Corresponding author. Tel.: +1 301210 6406; fax: +1 3012104699.

E-mail address: saura.sahu@fda.hhs.gov (S.C. Sahu).
}

The most prominent medicinal use of Creosote bush is for its antioxidant effects (Sheikh et al., 1997; Arteaga et al., 2005). It is generally recognized that the natural antioxidant NDGA is responsible for the medicinal value of this desert shrub (Robinson and Kwang-Jin, 1996; Zhang et al., 1999). Also NDGA was used as an antioxidant food preservative for fats and butter (Yamamoto et al., 1970), but it is no longer used for this purpose because of its toxicity.

Liver, the primary organ involved in xenobiotic metabolism, is a major target organ of many chemicals and drugs (Treinen-Molsen, 2001). Therefore, the potential for liver injury is one of the most serious safety concerns for drugs, food additives, food contaminants, dietary supplements and food-borne microbial pathogens. 
In vivo animal studies for assessing hepatotoxic potential of test materials of interest are expensive and time consuming. Therefore, alternate in vitro models are important to complement and/or supplement the animal studies, although relevant, for toxicological risk assessment (Green et al., 2001; MacGregor et al., 2001). The Food and Drug Administration is mandated by the NIH Revitalization Act of 1993 (P.L. No. 103-43) in concert with the ICCVAM Authorization Act of 2000 (P.L. No. 106-545) for federal regulatory agencies to: "(a) establish criteria for the validation and regulatory acceptance of alternative testing methods; and (b) recommend a process through which scientifically validated alternative methods can be accepted for regulatory use". Criteria for validation of alternative toxicological test methods have been published (ICCVAM, 1997). It has been shown that in vitro cytotoxicity is a useful predictor for estimating starting doses for in vivo acute toxicity studies, potentially reducing the number of animals used for such studies (Federal Register, 2001).

Hepatocytes and hepatocyte cell lines in culture retain many metabolic enzymes characteristic of the intact liver in vivo (Hengstler et al., 2000, 2002; Runge et al., 2001). They thus represent an excellent in vitro model for studying liver function, xenobiotic metabolism, pharmacology and toxicology. Cultured hepatocytes are widely used for the evaluation of liver functions and toxicity of chemicals, drugs and microbes (Barsig et al., 1998; Castell et al., 1997; Li et al., 1997; Michalopoulos, 1999).

The clone- 9 rat hepatocytes isolated from the normal liver of a 4-week-old Sprague-Dawley rat are used as an in vitro model for hepatotoxicity testing of chemicals and drugs (Barhoumi and Burghardt, 1996; Barhoumi et al., 2000, 2002; Barros et al., 2001; Garcia-Caballero et al., 2001; Grune et al., 2001, 2002; Hutchinson et al., 1998; Liegro et al., 2000; Neerman et al., 2004; Reeves et al., 2001; Thomspson et al., 1998). We tested this in vitro model system to assess the hepatotoxic potential and pro-oxidant activity of NDGA.

We have previously shown that flavonoids, a class of natural polyphenolic compounds, can act as double-edged biological swords both as anti- and prooxidants depending on their concentration and biological environment (Sahu et al., 2001). We hypothesized that the polyphenolic natural antioxidant NDGA might behave like flavonoids and demonstrate prooxidant activity. Therefore, the objective of this study was to assess the prooxidant activity along with the associated toxicity of NDGA in clone-9 rat hepatocyte cultures.

\section{Materials and methods}

\subsection{Materials}

Dulbecco's modified Eagle's medium (DMEM), Hanks' balanced salt solution (HBSS), HEPES, L-glutamine, and trypan blue $(0.4 \%)$ were purchased from Invitrogen Corporation (Grand Island, NY). Fetal bovine serum (FBS) was purchased from the Hyclone Labs (Logan, UT). Ethidium bromide, ethylene glycol-bis( $\beta$-amino-ethylether) $N, N, N^{\prime}, N^{\prime}$-tetra- acetic acid (EGTA) and NDGA were purchased from Sigma Chemical Co. (St. Louis, MO). The purity of NDGA was $>97 \%$. Thiobarbituric acid and trichloroacetic acid were purchased from Aldrich Chemical Co. (Milwaukee, WI). Clone-9 rat hepatocytes were obtained from American Type Culture Collection (ATCC, Manassas, VA).

\subsection{Hepatocyte cultures}

Clone-9 rat hepatocytes were cultured (Barros et al., 2001; Liegro et al., 2000) in Dulbecco's modified Eagle's medium (DMEM) containing $10 \%$ fetal bovine serum in $75 \mathrm{~cm}^{2}$ flasks at $37^{\circ} \mathrm{C}$ in a humidified atmosphere of $5 \% \mathrm{CO}_{2}$ in air as recommended by the ATCC.

\subsection{Treatment of hepatocyte cultures}

Hepatocyte cultures were trypsinized, suspended in the culture medium and seeded onto 6-well culture plates $24 \mathrm{~h}$ before the treatment with the test material. The plates were incubated at $37^{\circ} \mathrm{C}$ in a humidified atmosphere of $5 \% \mathrm{CO}_{2}$ in air. The seeded hepatocytes were washed with Hanks' balanced salt solution (HBSS) and the culture medium without FBS and phenol red was added to the cells. The freshly prepared test material dissolved in dimethyl sulfoxide (DMSO) was added to the hepatocyte cultures. The cultures with the test material were incubated at $37^{\circ} \mathrm{C}$ for $2 \mathrm{~h}$ in $5 \% \mathrm{CO}_{2}$ with controls containing equal volume of DMSO but without the test material. The test concentrations of NDGA were selected based on its antioxidant effects (Robinson et al., 1990). The concentration of DMSO $(0.1 \%, v / v)$ in the culture medium has no effect (Robinson et al., 1990). After the treatment period, the control and treated hepatocytes were processed immediately for various assays.

\subsection{Analytical procedures}

2.4.1. Cell viability

Cell viability was determined by trypan blue dye exclusion.

\subsubsection{Cytotoxicity}

Leakage of cytoplasmic enzymes from injured cells into the surrounding incubation medium is an accepted index for membrane damage and is frequently used as an indicator of cytotoxicity (Barsig et al., 1998; Gomez-Lechon et al., 1988; Hill and Roth, 1998; Jiang and Acosta, 1993; Mithchell et al., 1980; Xu et al., 2003). Leakage of the liver enzyme lactate dehydrogenase (LDH) is very commonly used for measuring cytotoxicity of test agents. Cytotoxicity was measured spectrophotometrically as the activity of LDH leaked into the incubation medium (Mithchell et al., 1980; Jiang and Acosta, 1993; Sahu et al., 2001; Sahu and Sapienza, 2005) and was expressed as a percentage of the total LDH activity released from the cells treated with 5\% Triton X-100 (Barsig et al., 1998; Hill and Roth, 1998).

\subsubsection{Oxidative stress}

Oxidative stress in hepatocyte cultures was measured by the nitroblue tetrazolium (NBT) method (Bacsi et al., 2005; Becerra and Albesa, 2002; Mochida et al., 1992; Riss and Moravec, 2004) and was expressed as a percentage of the control.

\subsubsection{Peroxidation of membrane lipids}

Lipid peroxidation is one of the best known manifestations of oxidative stress-induced cell injury. Membrane lipid peroxidation was determined by the spectrophotometric thiobarbituric acid (TBA) method (Buege and Aust, 1978) as described previously (Sahu et al., 2001; Sahu and Sapienza, 2005). Briefly, a $0.5 \mathrm{~mL}$ aliquot of the cell suspension was added to $0.5 \mathrm{~mL}$ of the reaction medium containing TBA $(0.375 \%$, w/v) and trichloroacetic acid $(15 \%, \mathrm{w} / \mathrm{v})$ in $0.25 \mathrm{~N} \mathrm{HCl}$. The reaction mixture was heated in boiling water for $15 \mathrm{~min}$ for color development, cooled to room temperature, and centrifuged to remove insoluble materials. The absorbance of clear supernatant was measured at $535 \mathrm{~nm}$, and the MDA 
concentration was calculated using the molar extinction coefficient of $1.56 \times 10^{5}$ (Buege and Aust, 1978). The results were expressed as a percentage of the control.

\subsubsection{DNA double-strand breaks}

DNA strand-breaks were determined by measuring the decrease in double-stranded DNA content using the fluorometric alkaline DNA unwinding method of Birnboim (1990) as described previously (Sahu et al., 2001; Sahu and Sapienza, 2005). Briefly, an aliquot of the cell suspension was added to the alkaline DNA unwinding solution and DNA was allowed to unwind. Ethidium bromide $(1 \mu \mathrm{g} / \mathrm{mL})$ was added to the reaction mixture, fluorescence was measured $(520 \mathrm{~nm}$ excitation and $590 \mathrm{~nm}$ emission), and the remaining double-stranded DNA was calculated as a percentage of the total.

\subsubsection{Mitochondria function test}

Mitochondria function was evaluated spectrophotometrically by measuring the degree of mitochondrial reduction of tetrazolium salt 3-(4,5dimethylthiazol-2-yl)-2,5-diphenyltetrazolium bromide (MTT) to formazan by succinic dehydrogenase (Carmichael et al., 1987; Hussain and Frazier, 2003; Hussain et al., 2005). Briefly, the treated cells were washed and then incubated at $37{ }^{\circ} \mathrm{C}$ in culture medium containing $0.05 \%$ MTT for $15 \mathrm{~min}$. The medium was then removed and the colored formazan was extracted from the cells in acidified isopropanol. The solution was centrifuged at $5000 \mathrm{~g}$ for $5 \mathrm{~min}$, and the absorbance of the clear supernatants was read at $540 \mathrm{~nm}$. The results were expressed as a percentage of the control.

\subsubsection{Cell proliferation}

Cell proliferation rates were determined by the cytotoxicity test based on the 3-(4,5-dimethylthiazole-2yl)-2,5-dimethyltetrazolium bromide (MTT) assay (Chan et al., 2005; Chen and Li, 2005; Denizot and Lang, 1986; Jiang and Acosta, 1993; Minervini et al., 2004; Mosmann, 1983). Briefly, culture supernatant was gently removed from the multi-well culture plates and the hepatocytes were washed with HBSS. The cultures were incubated with $0.1 \%$ MTT solution $\left(1 \mathrm{~mL} /\right.$ well) for $2 \mathrm{~h}$ at $37{ }^{\circ} \mathrm{C}$ in $5 \%$ $\mathrm{CO}_{2}$. After the incubation period the MTT solution was carefully removed and isopropanol or DMSO was added to the precipitates. The culture plates were shaken at room temperature for $1 \mathrm{~h}$ to dissolve the precipitate. The solution was centrifuged at $5000 \mathrm{~g}$ for $5 \mathrm{~min}$, and the absorbance of the clear supernatants was read at $540 \mathrm{~nm}$. The results were expressed as a percentage of the control.

\subsection{Statistical analysis of the data}

The statistical significance of the data was assessed using analysis of variance (ANOVA). Differences between the vehicle control and treated parameters with $p \leqslant 0.05$ were considered statistically significant.

\section{Results and discussion}

Hepatic cytotoxicity, oxidative stress, lipid peroxidation, DNA double-strand breaks, mitochondrial function and cell proliferation were used as the biomarkers of hepatotoxicity (Griffin et al., 1996; Hasegawa et al., 1995; James et al., 1993; Parola et al., 1996; Shen et al., 1994; Ushakova et al., 1996; Videla et al., 1995).

A concentration-dependent increase in cytotoxicity as measured by the leakage of the liver enzyme LDH into the surrounding incubation medium was observed when clone9 rat hepatocyte cultures were treated with NDGA (Fig. 1).

A concentration-dependent increase in oxidative stress was observed in clone-9 rat hepatocyte cultures when treated with NDGA (Fig. 2). Oxidative stress is known to play an important role in the toxicity of various xenobiotics and is considered to be involved in the mechanism of toxicity for a wide variety of chemicals (Stacey and Klaassen, 1981).

NDGA increased oxidative cell injury in the clone-9 rat hepatocytes as evidenced by the peroxidation of membrane lipids and DNA double-strand breaks. The polyphenol induced a concentration-dependent increase in membrane lipid peroxidation (Fig. 3) and decrease in double-stranded DNA content (Fig. 1). Total double-stranded DNA is a surrogate marker of cell viability; so its break down

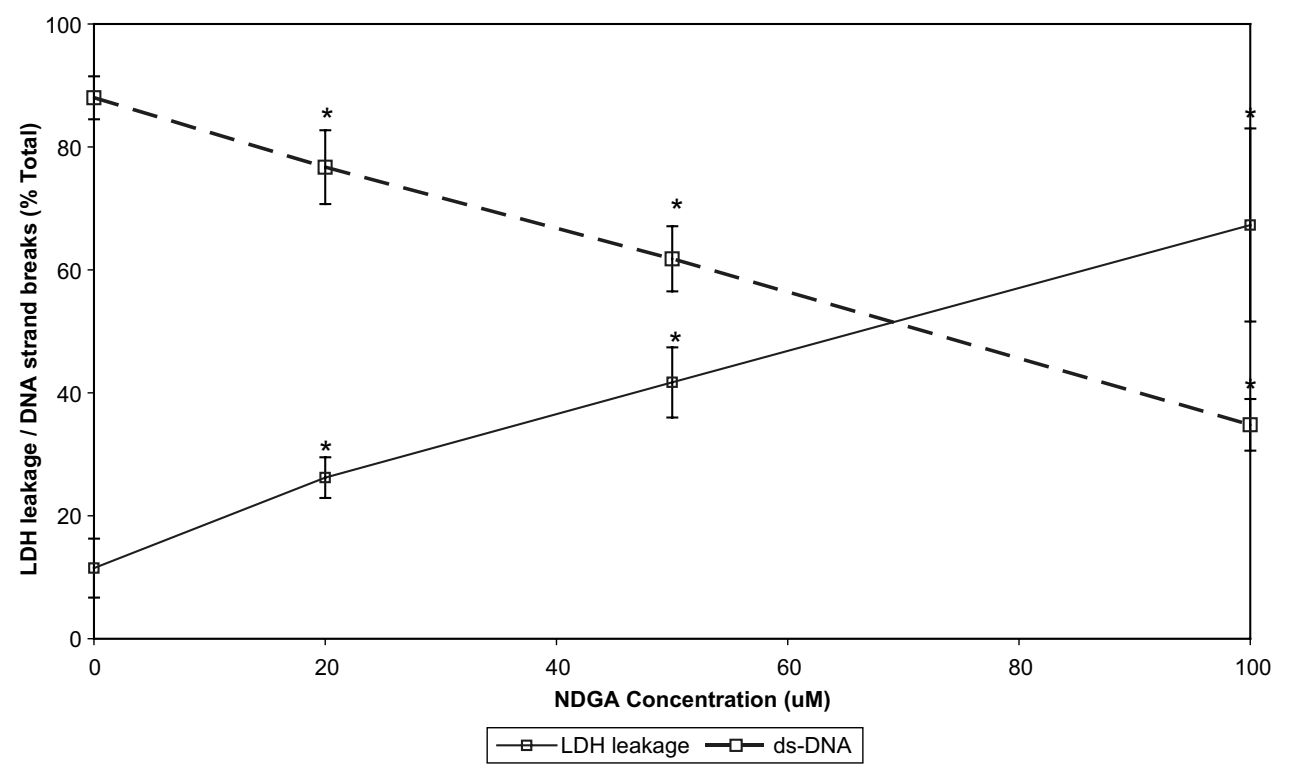

Fig. 1. LDH leakage and DNA strand breaks induced by NDGA in rat clone-9 hepatocyte cultures. Cytotoxicity (solid line) was measured by the leakage of the liver enzyme LDH into the culture medium and was expressed as a percentage of the total activity. Extent of DNA double-strand breaks (broken line) was expressed as a percentage of the total double-strands. Each value is the mean $\pm \mathrm{SD}$ of experiments from 10 independent hepatocyte cultures. * Significantly different from the control value $(p \leqslant 0.05)$. 


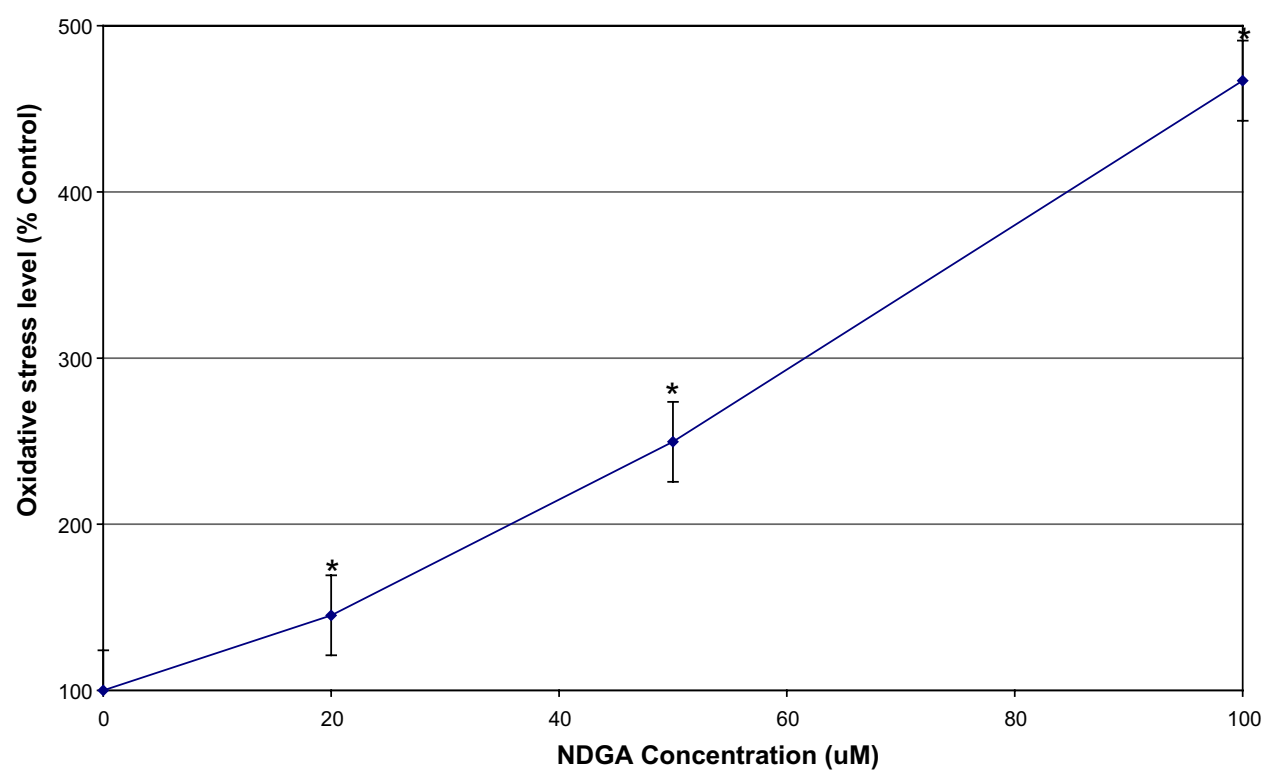

Fig. 2. Oxidative stress induced by NDGA in clone-9 rat hepatocyte cultures. Oxidative stress level was expressed as a percentage of the control. Each value is the mean $\pm \mathrm{SD}$ of experiments from 10 independent hepatocyte cultures. ${ }^{*}$ Significantly different from the control value $(p \leqslant 0.05)$.

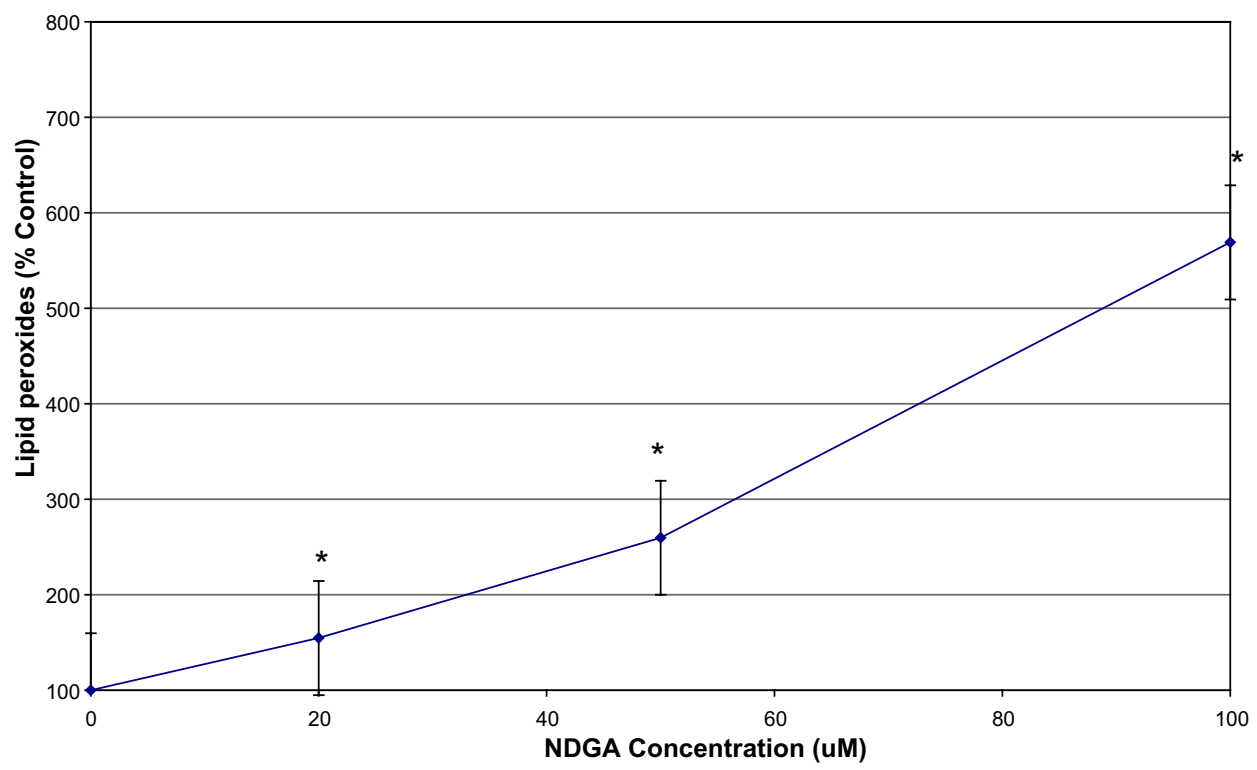

Fig. 3. Lipid peroxidation induced by NGDA in clone-9 rat hepatocyte cultures. Lipid peroxidation was expressed as a percentage of the control. Each value is the mean $\pm \mathrm{SD}$ of experiments from 10 independent hepatocyte cultures. ${ }^{*}$ Significantly different from the control value $(p \leqslant 0.05)$.

indicates cell death. Membrane lipid peroxidation and DNA double-strand breaks are considered to be manifestations of oxidative cell damage (Stacey and Klaassen, 1981; Griffin et al., 1996; Hasegawa et al., 1995; James et al., 1993; Parola et al., 1996; Shen et al., 1994; Ushakova et al., 1996; Videla et al., 1995).

A concentration-dependent decrease in mitochondrial function and cell growth was observed in clone-9 rat hepatocyte cultures treated with NDGA (Fig. 4).

The phenolic NDGA is an antioxidant (Robinson et al., 1990). It inhibits lipoxygenase activity (Kemal et al., 1987) and arachidonic acid release (Robinson et al., 1990). It suppresses cytotoxicity of hydroperoxides such as $t$-butylhydroperoxide (Nakayama et al., 1991) and hydrogen peroxide (Nakayama et al., 1992) to mammalian cells. Some antioxidants are known to prevent oxidative stress-induced toxicity. They are known to suppress mutagenicity and tumorigenicity believed to be induced by reactive oxygen species (ROS). Inhibition of tumor promotion by some antioxidants has been demonstrated (Emerit et al., 1983). The antimutagenic and antitumorigenic activities of NDGA have been reported (Avis et al., 1996; Biswal 


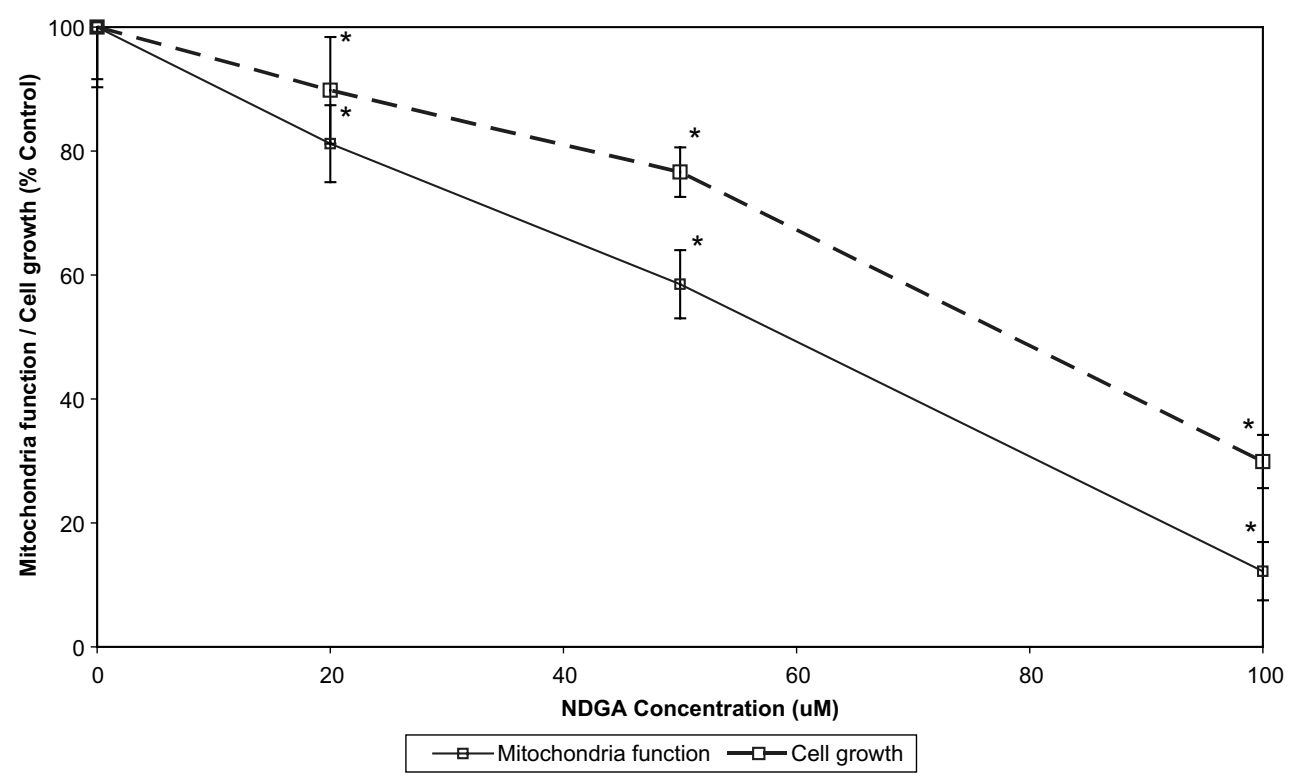

Fig. 4. Effect of NDGA on mitochondria function and cell growth in rat clone-9 hepatocyte cultures. Mitochondria function (solid line) and cell growth (broken line) was expressed as a percentage of the control. Each value is the mean \pm SD of experiments from 10 independent hepatocyte cultures. * Significantly different from the control value $(p \leqslant 0.05)$.

et al., 2000; Moody et al., 1998; Wang et al., 1991). Therefore, the beneficial effects of NDGA are believed to be due to its antioxidant properties. However, the results of our study presented here show that NDGA acts as a prooxidant in clone-9 rat hepatocyte cultures. It increased oxidative stress, oxidative cell injury and cytotoxicity. We have reported such toxic effects of polyphenolic flavonoids in primary rat hepatocytes (Sahu et al., 2001). Such adverse effects of supposedly beneficial antioxidants have been reported by other laboratories also (Galati et al., 2002). Phenolic antioxidants such as catechol, resorcinol, hydroquinone have been shown to be potential promoters of carcinogenesis in rat stomach (Shibata et al., 1990). It has been suggested that consumption of antioxidant herbal products can lead to hepatotoxicity (Stedman, 2002).

NDGA is known to cause cystic nephropathy in rats (Evan and Gardner, 1979; Gardner et al., 1987; Goodman et al., 1970; Grice et al., 1968). It has also been reported that prolonged use of the herbal product "Chaparral", prepared from the Creosote bush leaves, induces cystic renal disease (Smith, 1994), hepatitis (Katz and Saibil, 1990; Brent, 1999) and liver injury (Heron and Yarnell, 2001; Sheikh et al., 1997; Stickel et al., 2000) in humans. It has been suggested that NDGA is responsible for "chaparral" toxicity (Katz and Saibil, 1990).

In conclusion, NDGA exhibits adverse prooxidant effects on clone-9 rat hepatocyte cultures in the test concentration range of $20-100 \mu \mathrm{M}$, although it has been shown to have beneficial antioxidant effects on rat alveolar macrophages at a lower concentration range of less than $10 \mu \mathrm{M}$ (Robinson et al., 1990). Therefore, it would appear that this polyphenolic compound has the potential to act both as a pro- and antioxidant depending on its concentration and biological environment. In the cellular environment these two opposite effects may be competitive, and the cellular defense systems may modulate its toxicity.

\section{References}

Arteaga, S., Andrade-Cetto, A., Cardenas, R., 2005. Larrea tridentata (Creosote bush), an abundant plant of Mexican and US - American deserts and its metabolite nordihydroguaiaretic acid. J. Ethnopharmacol. 98, 139-231.

Avis, I.M., Boyle, T., Vos, M., Moody, T., Martinez, A., Mulshine, J., 1996. Growth control of lung cancer by interruption of 5-lipoxygenasemediated growth factor signaling. J. Clin. Invest. 97, 806-813.

Bacsi, A., Kannan, S., Lee, M., Hazra, T., Bodogh, I., 2005. Modulation of DNA- dependent protein kinase activity in chlorambucil-treated cells. Free Radical Biol. Med. 39, 1650-1669.

Barhoumi, R., Burghardt, R.C., 1996. Kinetic analysis of the chronology of patulin- and gossypol-induced cytotoxicity in vitro. Fund. Appl. Toxicol. 30, 290-297.

Barhoumi, R., Mouneimne, Y., Ramos, K., Phillips, T., Gupta, M., Burghardt, R.C., 2000. Analysis of benzo[a]pyrene partitioning and cellular homeostatis in a rat live cell line. Toxicol. Sci. 53, 264 270.

Barhoumi, R., Mouneimne, Y., Safe, S., Donnelly, K., Burghardt, R.C., 2002. Characterization of calcium oscillations in normal and benzo[a]pyrene-treated clone-9 cells. Toxicol. Sci. 68, 444-450.

Barros, L., Stutzin, A., Castro, J., Hetz, C., Hermosila, T., 2001. Nonselective cation channel as effectors of free-radical-induced rat liver cell necrosis. Hepatology 33, 114-122.

Barsig, J., Flesch, I., Kaufman, S., 1998. Hepatic cells as chemokine producers in murine listeriosis. Immunobiology 199, 87-104.

Becerra, M.C., Albesa, I., 2002. Oxidative stress induced by ciprofloxacin in Staphylococcus aureus. Biochem. Biophys. Res. Commun. 297, 1003-1007.

Birnboim, H.C., 1990. Fluorometric analysis of DNA unwinding to study strand breaks and repair in mammalian cells. Methods Enzymol. 186, $550-555$.

Biswal, S.S., Datta, K., Shaw, S., Feng, X., Tobertson, J., Kehrer, J., 2000. Glutathione oxidation and mitochondrial depolarization as mechanism 
of nordihydroguaiaretic acid-induced apoptosis in lipoxygenase-deficient FL5.12 cells. Toxicol. Sci. 53, 77-86.

Brent, J., 1999. Three new herbal hepatotoxic syndromes. J. Toxicol. Clin. Toxicol. 37, 715-719.

Buege, J.A., Aust, S.D., 1978. Microsomal lipid peroxidation. Methods Enzymol. 52, 302-310.

Carmichael, J., DeGraff, W., Gazder, A., MInna, J., Mitchell, J., 1987. Evaluation of a tetrazolium-based semiautomated colorimetic assay. Cancer Res. 47, 936-942.

Castell, J., Gomez-Leochon, M., Ponsoda, X., Bort, R., 1997. Use of cultured hepatocytes to investigate mechanisms of drug hepatotoxicity. Cell Biol. Toxicol. 13, 331-338.

Chan, J., Siu, K., Fung, K., 2005. Effect of arsenic trioxide on multidrug resistant hepatocellular carcinoma cells. Cancer Lett. 227, 153-162.

Chen, P., Li, J., 2005. Chemopreventive effect of punicalagin, a novel tannin component isolated from Terminalia catappa on H-ras-transformed NIH3T3 cells. Toxicol. Lett. 155, 259-267.

Denizot, F., Lang, R., 1986. Rapid colorimetric assay for cell growth and survival: modifications to the tetrazolium dye procedure giving improved sensitivity and reliability. J. Immunol. Methods 89, 271-277.

Emerit, I., Levy, A., Cerytti, P., 1983. Suppression of tumor promoter phorbolmyristate acetate-induced chromosome breakage by antioxidants and inhibitors of arachidonic acid metabolism. Mutat. Res. 110, 327-335.

Evan, A.P., Gardner, K.D., 1979. Nephron obstruction in nordihydroguaiaretic acid-induced renal cystic disease. Kidney Int. 15, 7-19.

Federal Register, 2001. In vitro methods for assessing acute systemic toxicity, vol. 66, no. 189, September 28, 2001, pp. 49686-49687.

Galati, G., Sabzevari, O., Wilson, J.X., O’Brien, P.J., 2002. Prooxidant activity and cellular effects of the phenoxyl radicals of dietary flavonoids and other polyphenolics. Toxicology 177, 91-104.

Garcia-Caballero, A., Olivares-Reyes, J., Catt, K., Garcia-Sainz, J., 2001. Angiotentsis AT receptor phosphorylation and desensitization in a hepatic cell line. Mol. Pharmacol. 59, 576-585.

Gardner, K.D., Reed, W., Evans, A., Hylarides, M., Leone, A., 1987. Endotoxin provocation of experimental renal cystic disease. Kidney Int. 32, 329-334.

Gomez-Lechon, M.J., Montoya, A., Lopez, P., Donato, T., Larrauri, A., Casell, J.V., 1988. The potential use of cultured hepatocytes in predicting hepatotoxicity of xenobiotics. Xenobiotica 18, 725-735.

Goodman, T., Grice, H., Becking, G., Salem, F., 1970. A cystic nephropathy induced by nordihydroguaiaretic acid in the rat. Lab. Investigat. 23, 93-107.

Green, S., Goldberg, A., Zurlo, J., 2001. Test smart - high production volume chemicals: an approach to implementing alternatives into regulatory toxicology. Toxicol. Sci. 63, 6-14.

Grice, H.C., Becking, G., Goodman, T., 1968. Toxic properties of nordihydroguaiaretic acid. Food Cosmet. Toxicol. 6, 155-1161.

Griffin, R.J., Dudley, C.N., Cunningham, M.L., 1996. Biochemical effects of mouse hepatocarcinogen Oxazepam: similarities to phenobarbital. Fund. Appl. Toxicol. 29, 147-154.

Grune, T., Klotz, L., Gieche, J., Rudeck, M., Sies, H., 2001. Protein oxidation and proteolysis by nonradical oxidants. Free Rad. Biol. Med. 30, 1243-1253.

Grune, T., Reinheckel, T., Li, R., North, J., Davies, K., 2002. Proteosome-dependent turnover of protein disulfide isomerase in oxidatively stressed cells. Arch. Biochem. Biophys. 397, 407-413.

Hasegawa, R., Chujo, T., Sai-Kato, K., Tanimura, A., Kurokawa, Y., 1995. Preventive effects of green tea against liver oxidative DNA damage and hepatotoxicity in rats treated with 2-nitropropane. Food Chem. Tox. 33, 961-970.

Hengstler, J., Ringael, M., Biefang, K., et al., 2000. Culture with cryopreserved hepatocytes: applicability for studies of enzyme induction. Chemico-Biol. Interact. 125, 51-73.

Hengstler, J., Utesch, D., Steinberg, P., et al., 2002. Cryopreserved primary hepatocytes as a constantly available in vitro model for the evaluation of human and animal drug metabolism and enzyme induction. Drug. Metab. Rev. 32, 81-118.
Heron, S., Yarnell, E., 2001. The safety of low dose Larrea tridentata (DC) Coville (creosote bush or chaparral): a retrospective clinical study. J. Alternat. Compliment. Med. 7, 175-185.

Hill, D.A., Roth, R.A., 1998. Naphthylisothiocyanate causes neutrophils to release factors that are cytotoxic to hepatocytes. Toxicol. Appl. Pharamacol. 148, 169-175.

Hussain, S.M., Frazier, J.M., 2003. Involvement of apoptosis in hydrazine induced toxicity in rat primary hepatocytes. Toxicol. In Vitro 17, 343 355.

Hussain, S.M., Hess, K., Gearhart, J., Geiss, K., Schlager, J., 2005. In vitro toxicity of nanoparticles in BRL $3 \mathrm{~A}$ rat liver cells. Toxicol. In Vitro 19, 63-68.

Hutchinson, R., Barhoumi, R., Miles, J., Burghardt, R.C., 1998. Attenuation of gossypol cytotoxicity by cyclic AMP in rat liver cell line. Toxicol. Appl. Pharmacol. 151, 311-318.

ICCVAM, 1997. Validation and regulatory acceptance of toxicological test methods, A Report of the ad hoc Interagency Coordinating Committee on the Validation of Alternative Methods. NIH Publication No. 97-3981, National Institute of Environmental Health Sciences, Research Triangle Park, North Carolina.

James, R.C., Harbison, R.D., Roberts, S., 1993. Phenylpropanolamine potentiation of acetaminophen-induced hepatotoxicity. Toxicol. Appl. Pharmacol. 118, 159-168.

Jiang, T., Acosta, D., 1993. An in vitro model of cyclosporine-induced nephrotoxicity. Fund. Appl. Toxicol. 20, 486-495.

Katz, M., Saibil, F., 1990. Herbal hepatitits: subacute hepatic necrosis secondary to chaparral leaf. J. Clin. Gastroenetrol. 12, 203-206.

Kemal, C., Louis-Flamber, P., Krupinski-Olsen, R., Shorter, A., 1987. Reductive inactivation of soyabin lipoxygenase by catechols: a possible mechanism for regulation of lipoxygenase activity. Biochemistry 26, 7064-7072.

Liegro, C., Bellafiore, M., Izquierdo, J., Rantanen, A., Cuezva, J., 2000. Untranslated regions of oxidative phosphorylation mRNA fuction in vivo as enhancers of translation. Biochem. J. 352, 109-115.

Li, A.P., Maurel, P., Gomez-Lechon, M., Leng, L., Jurima-Romet, M., 1997. Preclinical evaluation of drug-drug interaction potential. Chemico. Biol. Interact. 107, 5-16.

MacGregor, J.T., Collins, J.M., Sugiyama, Y., Tyson, C.A., Dean, J., 2001. In vitro human tissue models in risk assessment: report of a consensus-building workshop. Toxicol. Sci. 59, 17-36.

Michalopoulos, G., 1999. Hepatocyte cultures: currently used systems and their applications for studies of hepatocyte growth and differentiation. Tissue Eng. Methods Protocols 18, 227-243.

Minervini, F., Fornelli, F., Flynn, K.M., 2004. Toxicity and apoptosis induced by the mycotoxin nivalenol, deoxynivalenol and fumonisin B1 in human erythroleukemia cell line. Toxicol. In Vitro 18, 21-28.

Mithchell, D.B., Santone, K., Acosta, D., 1980. Evaluation of cytotoxicity in cultured cells by enzyme leakage. J. Tissue Culture Methods 6, 113116.

Mochida, S., Masaki, N., Ohta, Y., Matsui, A., Ogata, I., Fujiwara, K. 1992. In situ detection of oxidative stress in rat hepatocytes. J. Pathol. $167,83-89$.

Moody, T.W., Leyton, J., Martinez, A., Hong, S., Malkinson, A., Mulshine, J., 1998. Lipoxygenase inhibitors prevent lung carcinogenesis and inhibit non-small cell lung cancer growth. Exp. Lung Res. 24, 617-628.

Mosmann, T., 1983. Rapid colorimetric assay for cellular growth and survival: application to proliferation and cytotoxicity assays. J. Immunol. Methods 65, 55-63.

Nakayama, T., Hori, K., Terazawa, K., Kawakishi, S., 1991. Comparison of the cytotoxicity of different hydroperoxides to V79 cell. Free Rad. Res. Commun. 14, 173-178.

Nakayama, T., Hori, K., Ogawa, T., Kawashi, S., 1992. Suppression of hydrogen peroxide-induced mammalian cytotoxicity by nordihydroguaiaretic acid. Biosci. Biotechnol. Biochem. 56, 1162-1163.

Neerman, M.F., Zhang, W., Parrish, A., Simanek, E., 2004. In vitro and in vivo evaluation of a melamine dendrimer as a vehicle for drug delivery. Int. J. Pharm. 281, 129-132. 
Parola, M., Leonarduzzi, G., Robino, G., Poli, G., Dianzani, M., 1996. On the role of lipid peroxidation in the pathogenesis of liver damage. Free Rad. Biol. Med. 20, 351-359.

Reeves, W., Barhoumi, R., Lemke, S., McDonald, T., Phillips, T., Donnelly, K., 2001. Evaluation of methods for predicting toxicity of polycyclic aromatic hydrocarbon mixtures. Environ. Sci. Technol. 35, $1630-1636$.

Riss, T.L., Moravec, R.A., 2004. Use of multiple assay endpoints to investigate the effects of incubation time, dose of toxins and plating density in cell-based cytotoxicity assays. Assay Drug Develop. Technol. 2, 51-62.

Robinson, T., Kwang-Jin, K., 1996. Enhancement of epithelial Na, KATPase activity by $\mathrm{NO}_{2}$ and protective role of nordihydroguaiaretic acid. Am. J. Physiol. 270, L266-L272.

Robinson, T.W., Sevenian, A., Forman, H., 1990. Inhibition of arachidonic acid release by nordihydroguaiaretic acid and its antioxidant action in rat alveolar macrophages and Chinese hamster lung fibroblasts. Toxicol. Appl. Pharamacol. 105, 113-122.

Runge, D., Michalopoulos, G., Strom, S., Runge, D.M., 2001. Recent advances in human hepatocyte culture systems. Biochem. Biophys. Res. Commun. 274, 1-3.

Sahu, S.C., Sapienza, P.P., et al., 2005. Hepatotoxicity of androstenedione in pregnant rats. Food Chem. Toxicol. 43, 341-344.

Sahu, S.C., Flynn, T.J., Bradlaw, J.A., Roth, W.L., Barton, C.N., Yates, J.G., 2001. Pro-oxidant effects of the flavonoid myricetin on rat hepatocytes in culture. Toxicol. Methods 11, 277-283.

Sheikh, N., Philen, R., Love, L., 1997. Chaparral-associated hepatotoxicity. Arch. Int. Med. 157, 913-919.

Shen, H., Shi, C., Lee, H., Ong, C., 1994. Aflatoxin B 1 lipid peroxidation in rat liver. Toxicol. Appl. Pharmacol. 127, 145-150.

Shibata, M., Yamada, M., Hirose, M., Asakawa, E., Tatemasu, M., Ito, N., 1990. Early proliferative responses of forestamach and glandular stomach of rats treated with five different phenolic antioxidants. Carcinogenesis $11,425-429$
Smith, A., 1994. Cystic renal cell carcinoma and acquired renal cystic disease associated with consumption of chaparral tea: a case report. J. Urol. 152, 2089-2091.

Stacey, N.H., Klaassen, C.D., 1981. Inhibition of lipid peroxidation without prevention of cellular injury in isolated rat hepatocytes. Toxicol. Appl. Pharmacol. 58, 8-18.

Stedman, C., 2002. Herbal hepatotoxicity. Seminars in Liver Diseases 22, 195-206.

Stickel, F., Egerer, G., Seitz, H., 2000. Heptotoxicity of botanicals. Public Health Nutr. 3, 113-124.

Thomspson, D., Barhoumi, R., Burghardt, R., 1998. Comparative toxicity of eugenol and its quinine methide metabolite in cultured liver cells. Toxicol. Appl. Pharmacol. 149, 55-63.

Treinen-Molsen, M., 2001. Toxic responses of the liver. In: Klaassen, C.D. (Ed.), Casarett and Doull's Toxicology: The Basic Sciences of Poison. McGraw- Hill Professional, New York, pp. 471-490.

Ushakova, T., Melkonyan, H., Mudrik, N., Zhukova, A., Gaziev, A., Bradbury, R., 1996. The effect of dietary supplement on gene expression in mice. Free Rad. Biol. Med. 20, 279-284.

Videla, L.A., Smok, G., Simon, K., Junqueira, V., Fernandez, V., 1995. Influence of hyperthyroidism on lindane-induced hepatotoxicity in the rat. Biochem. Pharamacol. 50, 1557-1565.

Wang, Z.Y., Agarwal, R., Zhou, Z., Bickers, D., Mukhtar, H., 1991. Antimutagenic and antitumorigenic activities of nordihydroguaiaretic acid. Mutat. Res. 261, 153-162.

Xu, J., Ma, M., Purcell, W., 2003. Characterization of some cytotoxic endpoints using rat liver and HepG2 spheroids as in vitro models and their application in hepatotoxicity studies. Toxicol. Appl. Pharmacol. 189, 100-111.

Yamamoto, K., Murata, M., Nakano, H., 1970. Antioxidant therapy, Larrea, and livestock. Agr. Biol. Chem. 34, 1162-1168.

Zhang, L., Cosma, G., Gardner, K., Starks, K., Shi, X., Vallyathan, V., 1999. Scavenging of superoxide anion by chaparral. Mol. Cell. Biochem. 196, 157-161. 